\title{
A Voltammetric Sensor Based on NiO Nanoparticle-Modified Carbon-Paste Electrode for Determination of Cysteamine in the Presence of High Concentration of Tryptophan
}

\author{
Hassan Karimi-Maleh, ${ }^{1}$ Maryam Salimi-Amiri, ${ }^{2}$ Fatemeh Karimi, ${ }^{1}$ \\ Mohammad A. Khalilzadeh, ${ }^{1}$ and Mehdi Baghayeri ${ }^{3}$ \\ ${ }^{1}$ Department of Chemistry, Science and Research Branch, Islamic Azad University, Mazandaran, Iran \\ ${ }^{2}$ Department of Physics, Sari Branch, Islamic Azad University, Sari, Iran \\ ${ }^{3}$ Department of Chemistry, Faculty of Science, Hakim Sabzevari University, P.O. Box 397, Sabzevar, Iran \\ Correspondence should be addressed to Hassan Karimi-Maleh; h.karimi.maleh@gmail.com \\ and Fatemeh Karimi; fkm024@gmail.com
}

Received 25 May 2013; Accepted 8 September 2013

Academic Editor: Yehia Mechref

Copyright (C) 2013 Hassan Karimi-Maleh et al. This is an open access article distributed under the Creative Commons Attribution License, which permits unrestricted use, distribution, and reproduction in any medium, provided the original work is properly cited.

A carbon-paste electrode modified with ferrocenecarboxaldehyde and $\mathrm{NiO}$ nanoparticle (NiO/NPs) was used for the sensitive and selective voltammetric determination of cysteamine in the presence of tryptophan. The oxidation of cysteamine at the modified electrode was investigated by cyclic voltammetry (CV), chronoamperometry, and square-wave voltammetry (SWV). The values of the catalytic rate constant $\left(k_{h}\right)$ and diffusion coefficient $(D)$ for cysteamine were calculated. The modified electrode exhibits an efficient electron-mediating behavior together with well-separated oxidation peaks for cysteamine and tryptophan. At the optimum $\mathrm{pH}$ of 7.0 in a $0.1 \mathrm{M}$ phosphate buffer solution, the SWV anodic peak currents showed a linear relationship versus cysteamine concentrations in the range of $0.09-300.0 \mu \mathrm{M}$ and a detection limit of $0.06 \mu \mathrm{M}$. Finally, the proposed method was also examined as a selective, simple, and precise electrochemical sensor for the determination of cysteamine in real samples such as urine and capsule.

\section{Introduction}

Cysteamine is a significant thiol drug for the treatment of cystinosis [1]. Since cysteamine therapy has become available for patients with nephropathic cystinosis (MIM219800) in the early 1980s, quality of life for these patients has greatly improved $[2,3]$. The most frequent and most severe form, infantile cystinosis, is characterized by the development of renal Fanconi syndrome in the first year of life and leads to end-stage renal disease in the first decade of life when untreated [4]. Mutations in the CTNS gene, encoding for lysosomal cystine transporter cystinosin, are the causes of cystinosis [5]. Lysosomal accumulation of cystine, which is the hallmark of this autosomal recessive disorder, can be depleted by the aminothiol cysteamine [6]. Although treatment with cysteamine substantially decreases intracellular cystine accumulation, renal Fanconi syndrome is not cured, but end-stage renal disease can be postponed in the majority of the patients. Numerous chemical and instrumental techniques for detection of cysteamine have been reported [7-11].

Tryptophan (Trp), a vital constituent of proteins and a precursor for biologically important molecules such as the neurotransmitter serotonin and the neurohormone melatonin, is an amino acid essential to humans and animals due to its physiological roles [12]. It is also indispensable in human nutrition for establishing and maintaining a positive nitrogen balance [2]. In previous reports, spectroscopy [13], chemiluminescence [14], capillary electrophoresis [15], highperformance liquid chromatography [16], and electroanalysis [17-19] have been developed for tryptophan determination. Among these methods, electrochemical techniques, with high sensitivity, high accuracy, and simple operation, have 
been paid much more attention in the recent years [20-26]. However, the direct oxidation of Trp on the bare electrode is not attractive owing to sluggish electron-transfer processes and high overpotential [27].

In the recent years, nanostructured materials have also been incorporated into electrochemical sensors for environmental, biological, and pharmaceutical analyses [28-31]. While they have many properties similar to other types of bulk materials, they offer unique advantages including enhanced electron transfer, large edge plane/basal plane ratios, and rapid kinetics of the electrode processes [32-35].

Cysteamine is the simplest stable aminothiol and a degradation product of the amino acid, cysteine. On the other hand, tryptophan is an essential amino acid that has the same overpotential and the same overall structure of cysteamine. Therefore, it is necessary to develop a sensitive and selective method to measure each of those compounds separately and/or simultaneously. In this study, we have used voltammetric techniques to study the electrochemical behavior of cysteamine and tryptophan at an $\mathrm{NiO} / \mathrm{NPs}$ paste electrode modified with ferrocenecarboxaldehyde (FCAD/NiO/NPs/CPE). We reported a $p$ aminophenol-multiwall carbon nanotubes paste electrode for simultaneous determination of these compounds in the previous work. Compared to the previous report, this modified electrode has the best dynamic range, limit of detection, and good stability for determination of cysteamine in the presence of tryptophan.

\section{Experimental}

2.1. Chemicals. All chemicals used were of analytical reagent grade purchased from Merck (Darmstadt, Germany) unless otherwise stated. Doubly distilled water was used throughout.

Cysteamine and tryptophan were purchased from Merck and ferrocenecarboxaldehyde from Aldrich.

$1.0 \times 10^{-2} \mathrm{~mol} \mathrm{~L}^{-1}$ cysteamine solution was prepared daily by dissolving $0.08 \mathrm{~g}$ cysteamine in water, and the solution was diluted to $100 \mathrm{~mL}$ with water. The solution was kept in the refrigerator at $4^{\circ} \mathrm{C}$ in the dark. More diluted solutions were prepared by serial dilutions with water.

$1.0 \times 10^{-3} \mathrm{~mol} \mathrm{~L}^{-1}$ tryptophan solution was prepared daily by dissolving $0.02 \mathrm{~g}$ tryptophan in a buffer solution, $\mathrm{pH}$ 7.0, in a $100 \mathrm{~mL}$ volumetric flask, and under ultrasonication for several minutes. More diluted solutions were prepared by serial dilutions with water.

Phosphate buffer (sodium dihydrogen phosphate and disodium monohydrogen phosphate plus sodium hydroxide, $0.1 \mathrm{~mol} \mathrm{~L}^{-1}$ ) solutions (PBS) with different $\mathrm{pH}$ values were used.

Graphite powder (particle size $<50 \mu \mathrm{m}$ ) and highviscosity paraffin oil (density $=0.88 \mathrm{Kg} \mathrm{L}^{-1}$ ) from Fluka were used for the preparation of the electrodes.

2.2. Apparatus. The composition, size, and structural properties of the prepared nanocomposites were characterized by some equipped analysis systems. X-ray powder diffraction studies were carried out using a STOE diffractometer with $\mathrm{Cu}-\mathrm{Ka}$ radiation $(k=1.54 \AA)$.

Square-wave voltammetry (SWV), cyclic voltammetry, and chronoamperometry were performed in an electroanalytical system, $\mu$-Autolab PGSTAT 12 , potentiostat/galvanostat connected to a three-electrode cell, Metrohm Model 663 VA stand linked with a computer (Pentium IV, $1200 \mathrm{MHz}$ ), and with Autolab software. A conventional threeelectrode cell assembly consisting of a platinum wire as an auxiliary electrode and an $\mathrm{Ag} / \mathrm{AgCl} / \mathrm{KCl}_{\text {sat }}$ electrode as a reference electrode was used. The working electrode was either a carbon-paste electrode (CPE), NiO/NPs-modified carbon-paste electrode (NiO/NPs/CPE), FCAD/CPE, or FCAD/NiO/NPs/CPE. A pH meter (Corning, Model 140) with a double-junction glass electrode was used to check the $\mathrm{pH}$ of the solutions.

2.3. Synthesis of NiO/NPs Nanoparticle. To prepare the $\mathrm{NiO} / \mathrm{NPs}$, in a typical experiment, a $0.5 \mathrm{M}$ aqueous solution of nickel nitrate $\mathrm{Ni}\left(\mathrm{NO}_{3}\right)_{2}$ and a $0.5 \mathrm{M}$ aqueous solution of sodium hydroxide $(\mathrm{NaOH})$ were prepared in distilled water. Then, the beaker containing $\mathrm{NaOH}$ solution was heated at the temperature of about $55^{\circ} \mathrm{C}$. The $\mathrm{Ni}\left(\mathrm{NO}_{3}\right)_{2}$ solutions were added drop wise (slowly for $1.5 \mathrm{~h}$ ) to the above heated solution under high-speed stirring. The beaker was sealed at this condition for $2 \mathrm{~h}$. The precipitated $\mathrm{NiO} / \mathrm{NPs}$ were cleaned with deionized water and ethanol then calcined at $300^{\circ} \mathrm{C}$ for 1.5 hours. Main reactions occuring during the experimental procedure can be written briefly as follows:

$$
\begin{aligned}
2 \mathrm{NaOH}_{(\mathrm{s})} & \longrightarrow 2 \mathrm{Na}^{+}{ }_{(\mathrm{aq})}+2 \mathrm{OH}^{-}{ }_{(\mathrm{aq})} \\
\mathrm{Ni}\left(\mathrm{NO}_{3}\right)_{2} \cdot 6 \mathrm{H}_{2} \mathrm{O}_{(\mathrm{s})} & \longrightarrow \mathrm{Ni}^{2+}{ }_{(\mathrm{aq})}+2 \mathrm{NO}_{3}^{-{ }_{(\mathrm{aq})}}+6 \mathrm{H}_{2} \mathrm{O}_{(\mathrm{aq})}
\end{aligned}
$$

$\mathrm{Ni}^{2+}{ }_{(\mathrm{aq})}+2 \mathrm{OH}^{-}{ }_{(\mathrm{aq})}+x \mathrm{H}_{2} \mathrm{O}_{(\mathrm{aq})} \longrightarrow \mathrm{Zn}(\mathrm{OH})_{2} \cdot x \mathrm{H}_{2} \mathrm{O}_{(\mathrm{s})} \downarrow$

$$
\begin{gathered}
\mathrm{Ni}(\mathrm{OH})_{2} \cdot x \mathrm{H}_{2} \mathrm{O}_{(\mathrm{s})} \stackrel{100}{\longrightarrow} \mathrm{Ni}(\mathrm{OH})_{2(\mathrm{~s})}+x \mathrm{H}_{2} \mathrm{O}_{(\mathrm{g})} \uparrow \\
\mathrm{Ni}\left(\mathrm{OH}_{2}\right)_{(\mathrm{s})} \stackrel{300}{\longrightarrow} \mathrm{NiO}_{(\mathrm{s})}+\mathrm{H}_{2} \mathrm{O}_{(\mathrm{g})} \uparrow
\end{gathered}
$$

2.4. Preparation of the Modified Electrode. $15.0 \mathrm{mg}$ of ferrocenecarboxaldehyde was hand mixed with $885 \mathrm{mg}$ of graphite powder and $100 \mathrm{mg}$ of $\mathrm{NiO} / \mathrm{NPs}$ in a mortar and pestle. Using a syringe, $0.45 \mathrm{~g}$ of paraffin was added to the mixture and mixed well for 75 min until a uniformly wetted paste was obtained. The paste was then packed into a glass tube. Electrical contact was made by pushing a copper wire down the glass tube into the back of the mixture. When necessary, a new surface was obtained by pushing an excess of the paste out of the tube and polishing it on a weighing paper. The unmodified carbon-paste electrode was prepared in the same way without adding mediator and $\mathrm{NiO} / \mathrm{NPs}$ to the mixture.

2.5. Preparation of Real Samples. Five cystagone $150 \mathrm{mg}$ capsules produced by Mylan Drug Company were completely 


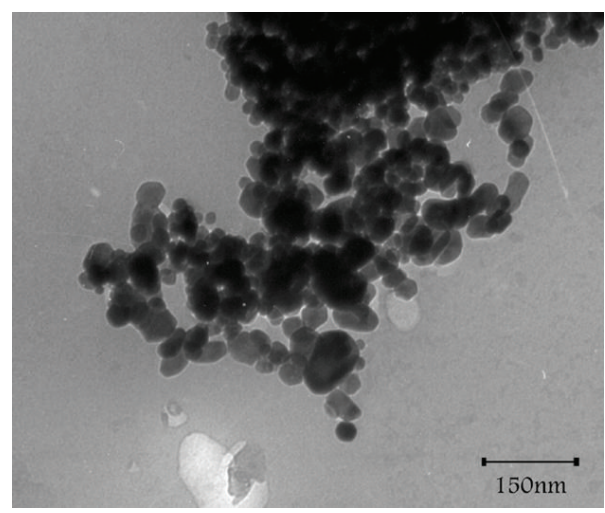

FIgURE 1: TEM image of NiO nanoparticles.

crushed and homogenized. Then, $10 \mathrm{mg}$ of the powders was accurately weighed and dissolved with ultrasonication in $100 \mathrm{~mL}$ of ethanol-water $(1: 2)$ solution. Then, different amount of the solution plus $10 \mathrm{~mL}$ of $0.10 \mathrm{~mol} \mathrm{~L}^{-1}$ buffer $(\mathrm{pH}$ 7.0) was used for the analysis.

The urine samples were obtained from the Sari Health Center and were stored in a refrigerator immediately after collection. Ten milliliters of the sample was centrifuged for $15 \mathrm{~min}$ at $2000 \mathrm{rpm}$. The supernatant was filtered using a $0.45 \mu \mathrm{m}$ filter and then diluted 5 times with universal buffer $\mathrm{pH}=7.0$. The solution was transferred into the voltammetric cell to be analyzed without any further pretreatment. Standard addition method was used for the determination of cysteamine in real samples.

\section{Results and Discussion}

3.1. Nanostructures Characterization. The XRD patterns of the $\mathrm{NiO}$ nanostructure showed diffraction peaks absorbed at $2 \theta$ values (Figure 1 ). The prominent peaks were used to calculate the grain size via the Scherrer equation expressed as follows:

$$
D=\frac{K \lambda}{\beta \cos \theta},
$$

where $\lambda$ is the wavelength $(\lambda=1.542 \AA)(\mathrm{Cu}-\mathrm{K} \alpha), \beta$ is the full width at half maximum (FWHM) of the line, and $\theta$ is the diffraction angle. The grain size of the $\mathrm{NiO}$ nanostructure was $15 \mathrm{~nm}$, and the peaks were observed at the (111), (200), (220), (311), and (222) planes. These peaks correspond to NiO.

The morphology of the as-grown nanostructures was characterized by TEM technique. Figure 1 shows the TEM images of the product synthesized. Figure 1 shows a typical TEM image of the NiO/NPs. The dark spots correspond to $\mathrm{NiO} / \mathrm{NPs}$, which were only synthesized in our synthesis condition. NPs with near-spherical shapes were synthesized. It is clear that, in this case, an $\mathrm{NiO}$ nanoparticle was successfully prepared.

3.2. Electrochemistry of FCAD/NiO/NPs/CPE. Ferrocenederivatives are known as good Ox/Red mediators with good electrochemical activity in the aqueous solution [36].

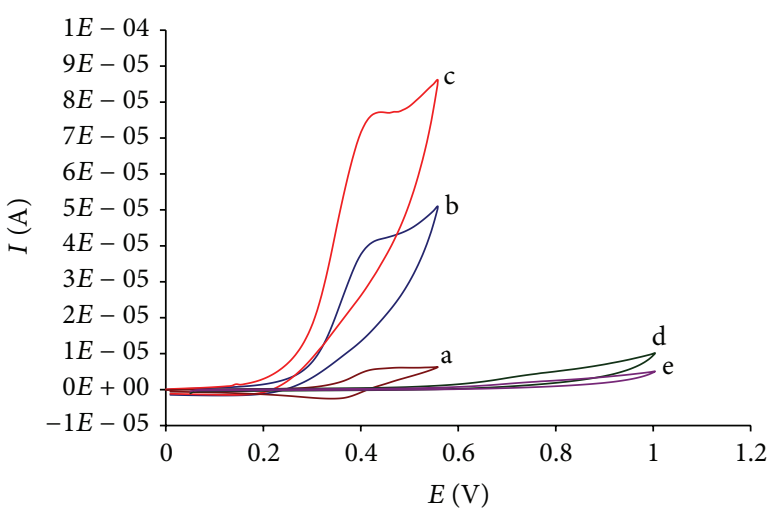

FIGURE 2: Cyclic voltammograms of (a) the buffer solution at $\mathrm{FCAD} / \mathrm{NiO} / \mathrm{NPs} / \mathrm{CPE}$; (b) $1.0 \mathrm{mM}$ cysteamine at FCAD/CPE; (c) $1.0 \mathrm{mM}$ cysteamine at FCAD/NiO/NPs/CPE; (d) $1.0 \mathrm{mM}$ cysteamine at $\mathrm{NiO} / \mathrm{NPs} / \mathrm{CPE}$; and (e) $1.0 \mathrm{mM}$ cysteamine at CPE. Conditions: $0.1 \mathrm{~mol} \mathrm{~L}^{-1} \mathrm{PBS}$ ( $\mathrm{pH} \mathrm{7.0)}$ ), scan rate of $20 \mathrm{mV} \mathrm{s}^{-1}$.

The electrochemical properties of the modified electrode were studied by cyclic voltammetry in the buffer solution ( $\mathrm{pH}$ 7.0). The experimental results show well-defined and reproducible anodic and cathodic peaks related to the ferrocenecarboxaldehyde/ferroceniumcarboxaldehyde redox coupled with quasireversible behavior (with peak separation potential being $\Delta E_{p}=E_{p a}-E_{p c}=80 \mathrm{mV}$ ) (Figure 2 curve a).

3.3. Electrocatalytic Investigation. In the first stage to test the potential electrocatalytic activity of the $\mathrm{FCAD} / \mathrm{NiO} / \mathrm{NPs} / \mathrm{CPE}$, its cyclic voltammetric responses at $20 \mathrm{mV} \mathrm{s}^{-1}$ were obtained in, $\mathrm{pH}$ 7.0, phosphate buffer in the absence and in the presence of $1.0 \mathrm{mM}$ cysteamine, and the data are presented in Figure 2. In the absence of cysteamine, a pair of well-defined redox peaks of $\mathrm{FCAD} / \mathrm{NiO} / \mathrm{NPs} / \mathrm{CPE}$ can be observed (Figure 2, curve a). Upon the addition of $1.0 \mathrm{mM}$ cysteamine, there was a drastic enhancement of the anodic peak current, and, in addition, no cathodic current was observed in the reverse scan (Figure 2, curve c). This behavior is consistent with a very strong electrocatalytic effect. Under the same experimental conditions, the direct oxidation of cysteamine at $\mathrm{NiO} / \mathrm{NPs} / \mathrm{CPE}$ and $\mathrm{CPE}$ shows an irreversible wave at more positive potentials (Figure 2, curves $\mathrm{d}$ and e). The catalytic peak potential is found to be about $420 \mathrm{mV}$, whereas that of the uncatalyzed peak is about $780 \mathrm{mV}$. Thus, a decrease in the overvoltage of approximately $360 \mathrm{mV}$ and an enhancement of the peak current are also achieved with the modified electrode. In a similar condition, at a surface of FCAD/CPE (curve b), cysteamine has a less current if compared to $\mathrm{FCAD} / \mathrm{NiO} / \mathrm{NPs} / \mathrm{CPE}$ in the same condition that is relative to the presence of $\mathrm{NiO} / \mathrm{NPs}$ at a surface of electrode with high conductivity in electrode matrix. On the basis of the information, we suggest the electrocatalytic mechanism in Scheme 1 for the oxidation of cysteamine at a surface of this sensor.

Figure 3 (inset) shows the voltammetric peaks potential of $\mathrm{FCAD} / \mathrm{NiO} / \mathrm{NPs} / \mathrm{CPE}$ at scan rates ranging from 4 to 


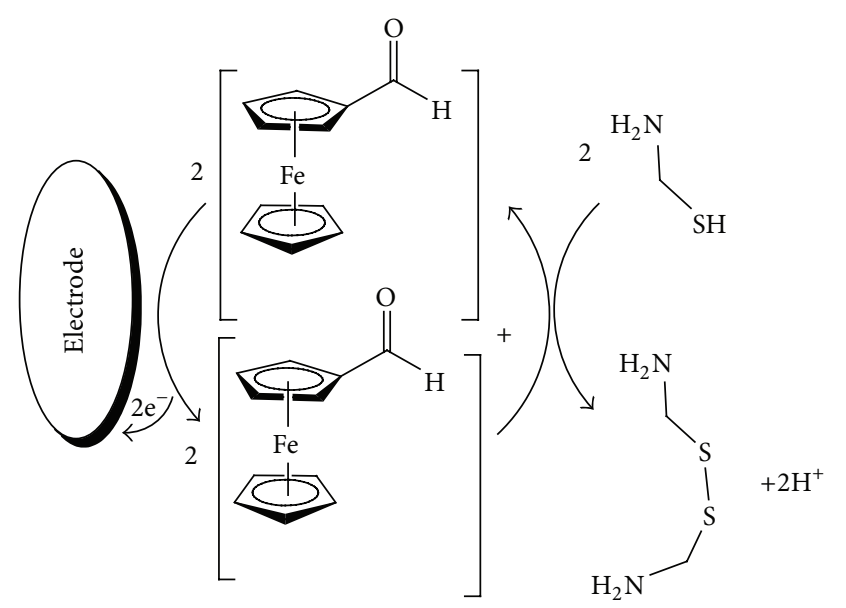

SCHEME 1

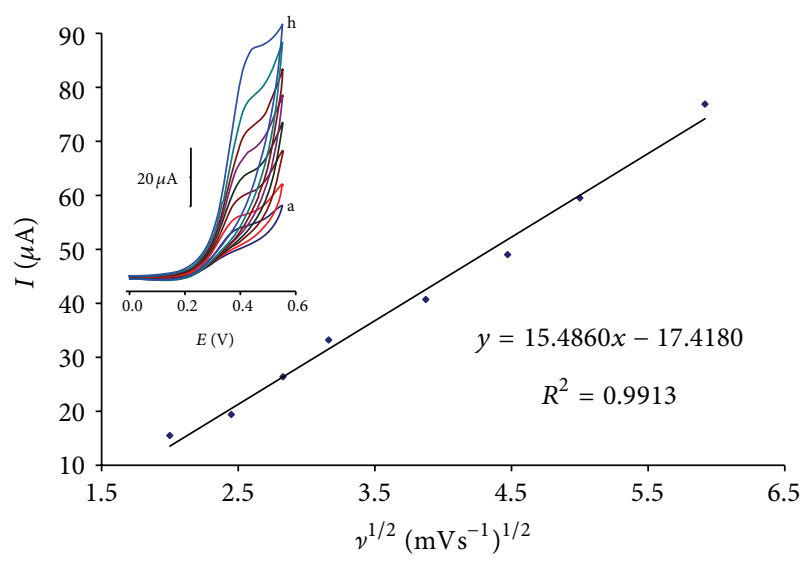

FIGURE 3: Plot of $I_{p a}$ versus $\nu^{1 / 2}$ for the oxidation of cysteamine at FCAD/NiO/NPs/CPE. Inset: (A) cyclic voltammograms of $0.5 \mathrm{mM}$ cysteamine at various scan rates: (a) 4; (b) 6; (c) 8; (d) 10; (e) 15; (f) 20; (g) 25, and (h) $35 \mathrm{mV} \mathrm{s}^{-1}$ in $0.1 \mathrm{~mol} \mathrm{~L}^{-1} \mathrm{PBS}$ ( $\mathrm{pH} \mathrm{7.0)}$.

$35 \mathrm{mV} \mathrm{s}^{-1}$ at $\mathrm{pH} 7.0$ containing $0.5 \mathrm{mM}$ cysteamine. We observed a linear variation of the peak current with the square root of scan rate $\left(v^{1 / 2}\right)\left(r^{2}=0.9913\right)$ (Figure 3$)$. This result clearly indicates a diffusion-controlled electrooxidative process.

To obtain information about the rate-determining step, the Tafel plot was drawn, as derived from points in the Tafel region of the cyclic voltammogram (Figure 4 ). The slope of the Tafel plot was equal to $2.3 R T / n(1-\alpha) F$, which came up to $0.1303 \mathrm{~V} /$ decade $^{-1}$ for scan rates of $20 \mathrm{mV} \mathrm{s}^{-1}$, respectively. Therefore, we obtained the mean value of $\alpha$ equal to 0.55 . In addition, the value of $\alpha$ was calculated for the oxidation of cysteamine at $\mathrm{pH} 7.0$ for both the modified and unmodified carbon nanotubes paste electrodes using the following equation [37]:

$$
\alpha n_{\alpha}=\frac{0.048}{E_{P}-E_{P / 2}},
$$

where $E_{P / 2}$ is the potential corresponding to $I_{P / 2}$. The values for $\alpha n_{\alpha}$ were found to be 0.54 and 0.11 at the surface

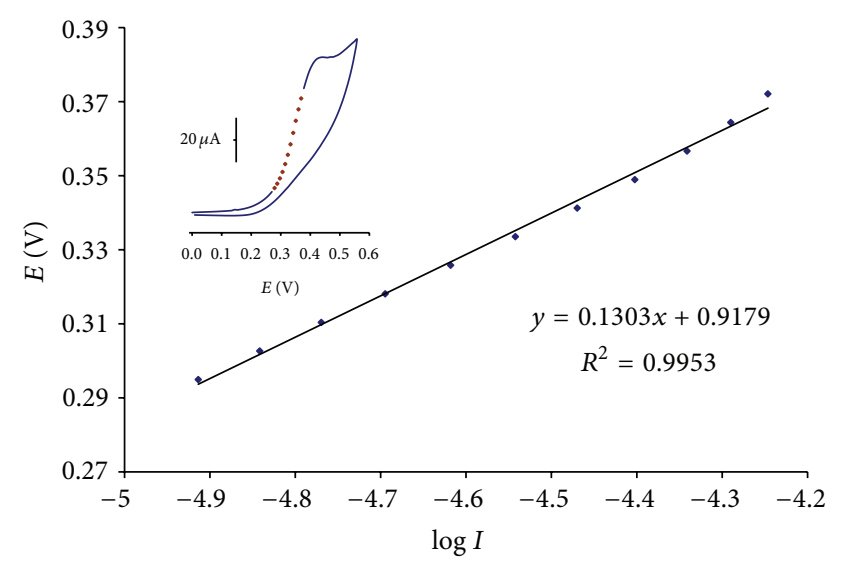

Figure 4: Tafel plot for FCAD/NiO/NPs/CPE in $0.1 \mathrm{~mol} \mathrm{~L}^{-1} \mathrm{PBS}$ ( $\mathrm{pH} 7.0$ ) with scan rates of $20 \mathrm{mV} \mathrm{s}^{-1}$ in the presence of $1.0 \mathrm{mM}$ cysteamine.

of both $\mathrm{FCAD} / \mathrm{NiO} / \mathrm{NPs} / \mathrm{CPE}$ and the $\mathrm{CPE}$, respectively. Those values show that the overpotential of cysteamine oxidation is reduced at the surface of $\mathrm{FCAD} / \mathrm{NiO} / \mathrm{NPs} / \mathrm{CPE}$ and also that the rate of electron-transfer process is greatly enhanced. This phenomenon is, thus, confirmed by the larger $I_{p a}$ values recorded during cyclic voltammetry at $\mathrm{FCAD} / \mathrm{NiO} / \mathrm{NPs} / \mathrm{CPE}$.

Chronoamperometric measurements of cysteamine at $\mathrm{FCAD} / \mathrm{NiO} / \mathrm{NPs} / \mathrm{CPE}$ were carried out at the working electrode potential of $500 \mathrm{mV}$ for various concentrations of cysteamine (Figure 5(A)). For an electroactive material (cysteamine in this case) with a diffusion coefficient of $D$, the current observed for the electrochemical reaction at the mass transport limited condition is described by the Cottrell equation. Experimental plots of $I$ versus $t^{-1 / 2}$ were employed, with the best fits for $0.3 \mathrm{mM}$ of cysteamine (Figure 5(B)). From the resulting slope and Cottrell equation, the mean value of the $D$ was found to be $2.4 \times 10^{-5} \mathrm{~cm}^{2} \mathrm{~s}^{-1}$.

Chronoamperometry can also be employed to evaluate the catalytic rate constant, $k_{h}$, for the reaction between cysteamine and the FCAD/NiO/NPs/CPE according to the method of Galus [38]:

$$
\frac{I_{C}}{I_{L}}=\prod^{1 / 2} \gamma^{1 / 2}=\prod^{1 / 2}\left(k C_{b} t\right)^{1 / 2}
$$

where $I_{C}$ is the catalytic current of cysteamine at the FCAD/NiO/NPs/CPE, $I_{L}$ is the limited current in the absence of cysteamine, and $t$ is the time elapsed (s). Based on the slope of the $I_{C} / I_{L}$ versus $t^{1 / 2}$ plot, $k_{h}$ can be obtained for a given cysteamine concentration (Figure $5(\mathrm{C})$ ). From the values of the slopes, an average value of $k_{h}$ was found to be $k_{h}=$ $1.5 \times 10^{5} \mathrm{~mol}^{-1} \mathrm{~L} \mathrm{~s}^{-1}$. The value of $k_{h}$ also explains the sharp feature of the catalytic peak observed for catalytic oxidation of cysteamine at the surface of FCAD/NiO/NPs/CPE.

3.4. Square-Wave Voltammetry Investigations. Since squarewave voltammetry, SWV, has a much higher current sensitivity and better resolution than cyclic voltammetry, it 


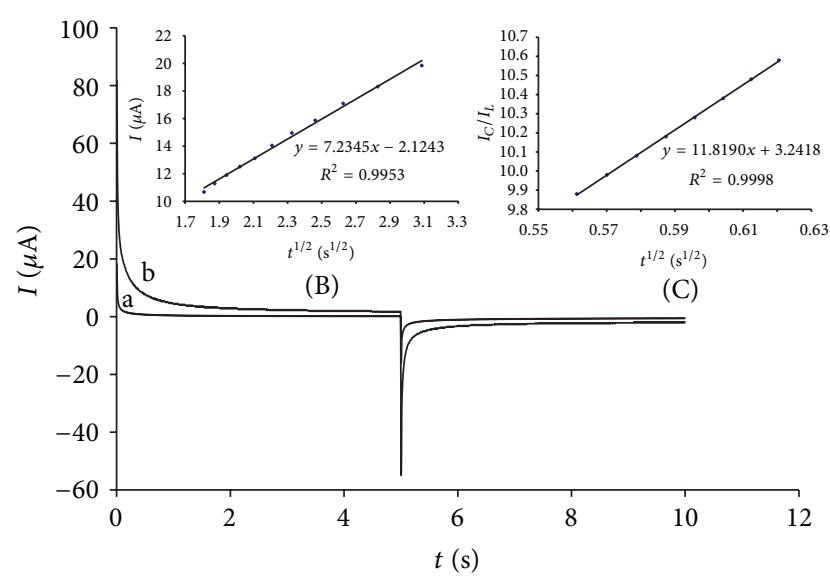

(A)

Figure 5: (A) Chronoamperograms obtained at $\mathrm{FCAD} / \mathrm{NiO} /$ $\mathrm{NPs} / \mathrm{CPE}$ (1) in the absence and in the presence of (2) $300.0 \mu \mathrm{M}$ cysteamine at $\mathrm{pH}$ 7.0. (B) Cottrell's plot for the data from the chronoamperogram. (C) Dependence of $I_{c} / I_{L}$ on the $t^{1 / 2}$ derived from the chronoamperogram data.

TABLE 1: Interference study for the determination of $10.0 \mu \mathrm{M}$ cysteamine under the optimized conditions.

\begin{tabular}{lc}
\hline Species & $\begin{array}{c}\text { Tolerant limits } \\
\left(W_{\text {Substance }} / W_{\text {Cysteamine }}\right)\end{array}$ \\
\hline $\begin{array}{l}\text { Glucose, fructose, lactose, and sucrose } \\
\text { Valine, methionine, glycine, lucine, } \\
\text { histidine, glutamic acid, alanine, glycine, } \\
\text { and phenylalanine }\end{array}$ & 9000 \\
$\mathrm{Li}^{+}, \mathrm{Cl}^{-}, \mathrm{CO}_{3}{ }^{2-}, \mathrm{ClO}_{4}{ }^{-}, \mathrm{SO}_{4}{ }^{2-}, \mathrm{SCN}^{-}, \mathrm{Na}^{+}$, & 800 \\
$\mathrm{Mg}^{2+}, \mathrm{K}^{+}$, and $^{+} \mathrm{Ca}^{+2}$ & 700 \\
Ascorbic acid & \\
Starch & \\
\end{tabular}

${ }^{*}$ After addition of $1 \mathrm{mM}$ ascorbic oxidase.

was used to estimate the lower limit of detection of cysteamine. Responses were linear with cysteamine concentrations ranging from 0.09 to $300 \mu \mathrm{M}$ and a current sensitivity of $0.274 \mu \mathrm{A} /(\mu \mathrm{mol} / \mathrm{L})$. The detection limit was determined at $0.06 \mu \mathrm{M}$ cysteamine according to the definition of $Y_{\mathrm{LOD}}=$ $Y_{B}+3 \sigma$.

The main objective of the present work was to develop a modified electrode that is capable of both electrocatalytic oxidation of cysteamine and separation of the electrochemical responses of cysteamine and tryptophan. As we know, peaks potentials for oxidation of cysteamine and tryptophan at an unmodified electrode are about 780 and $800 \mathrm{mV}$, respectively [39]. These results show, at unmodified $\mathrm{NiO} / \mathrm{NPs} / \mathrm{CPE}$, that the peaks potentials of cysteamine and tryptophan overlapped with each other [39]. On the other hand, at the modified electrode, these compounds have two wellseparated peaks potentials (with a $420 \mathrm{mV}$ separation of the peaks) (Figure 6 inset). Therefore, the modifier has a critical role, and it is necessary for determination of cysteamine and tryptophan simultaneously.

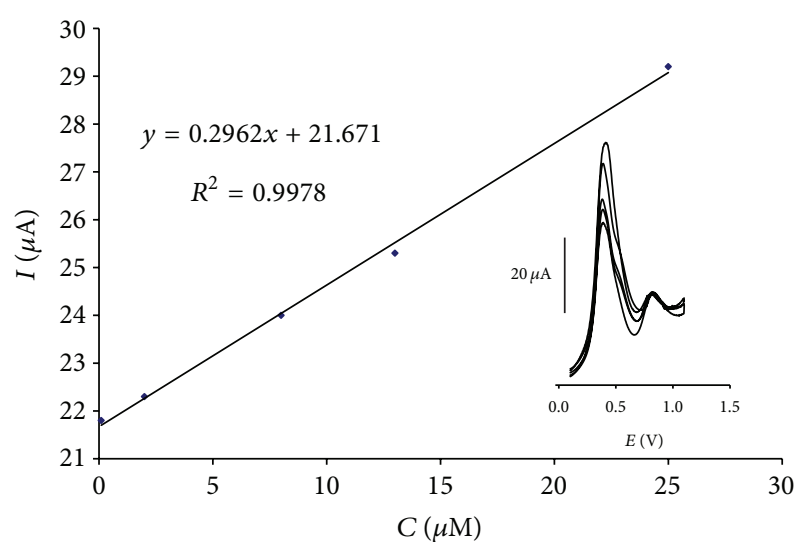

FIGURE 6: Plot of peak currents versus cysteamine concentration. Insert: square-wave voltammograms of cysteamine and tryptophan under the optimum conditions. $20.0 \mu \mathrm{M}$ tryptophan with cysteamine concentrations of (from inner to outer) (1) $0.1(2) ; 2.0$; (3) 8.0 ; (4) 13.0 ; and (5) $25.0 \mu \mathrm{M}$.

Figure 6 inset shows typical SWV for the simultaneous determination of cysteamine and tryptophan. Figure 6 shows the dependence of SWV peak currents on the concentration of cysteamine. The SW voltammetric results showed that simultaneous determination of cysteamine and tryptophan with two well-distinguished anodic peaks at 380 and $800 \mathrm{mV}$ potentials, corresponding to the oxidation of cysteamine and tryptophan, is possible at the modified electrode.

The sensitivities of the modified electrode towards the oxidation of cysteamine in the absence and presence of tryptophan were found to be 0.296 and $0.274 \mu \mathrm{A} / \mu \mathrm{M}$, respectively. It is interesting to note that the sensitivities of the modified electrode towards cysteamine in the absence and presence of tryptophan were virtually the same, which indicates that the oxidation processes of cysteamine and tryptophan at the modified electrode are independent and that simultaneous or independent measurements of the two compounds are, therefore, possible without any interference.

3.5. Interference Study. In order to evaluate the selectivity of the proposed method in cysteamine determination, the effects of various foreign species on the determination of $10.0 \mu \mathrm{M}$ cysteamine were investigated. The tolerance limit was taken as the maximum concentration of the foreign substances causing an approximately $\pm 5 \%$ relative error in the determination. The results are shown in Table 1.

3.6. Determination of $C A$ in Real Samples. In order to demonstrate the electrocatalytic oxidation of cysteamine in real samples, we examined the voltammetric determination of cysteamine in capsule and urine samples. The result was also compared with the standard method used for cysteamine determination. The proposed sensor was also compared with a published method [39] in real sample analysis, the results of which are given in Table 2 . The precision of the measurements was checked using the $F$-test method. As the results showed, the calculated $F$-values from the experimental data $\left(F_{\mathrm{ex}}\right)$ are 
TABLE 2: Determination of cysteamine in real samples $(n=3)$.

\begin{tabular}{|c|c|c|c|c|c|c|c|c|}
\hline Sample & Added $(\mu \mathrm{M})$ & Expected $(\mu \mathrm{M})$ & Founded $(\mu \mathrm{M})$ & Founded [39] $(\mu \mathrm{M})$ & $F_{\mathrm{ex}}$ & $F_{\text {tab }}$ & $t_{\mathrm{ex}}$ & $\overline{t_{\mathrm{tab}}}$ \\
\hline \multirow{3}{*}{ Capsule } & - & 10.00 & $9.88 \pm 0.32$ & $10.25 \pm 0.45$ & 6.5 & 19 & 1.9 & 3.8 \\
\hline & 10.00 & 20.00 & $20.55 \pm 0.56$ & $20.68 \pm 0.75$ & - & - & & - \\
\hline & 5.00 & 25.00 & $24.95 \pm 0.22$ & $25.55 \pm 0.50$ & - & - & & - \\
\hline \multirow{3}{*}{ Urine $^{\mathrm{a}}$} & - & - & $<\mathrm{LOD}$ & $<$ LOD & - & - & & - \\
\hline & 15.00 & 15.00 & $15.65 \pm 0.75$ & $15.87 \pm 1.00$ & 8.9 & 19 & 2.3 & 3.8 \\
\hline & 10.00 & 25.00 & $25.79 \pm 0.89$ & $25.95 \pm 1.04$ & - & - & & - \\
\hline Urine $^{\mathrm{b}}$ & - & - & $5.55 \pm 0.45$ & $5.67 \pm 0.84$ & 7.5 & 19 & 2.1 & 3.8 \\
\hline
\end{tabular}

less than the values obtained from the $F$-test table $\left(F_{\text {tab}}\right.$, $P=0.05)$ with the same degree of freedom. In addition, the accuracy of the obtained results by the proposed sensor was checked Student's $t$-test. As shown in Table 2, the measured $t$-values from the experimental data $\left(t_{\mathrm{ex}}\right)$ are less than the obtained $t$-values from Student's $t$-test table $\left(t_{\mathrm{tab}}, P=0.05\right)$ with the same degree of freedom. These results confirm that the precision and accuracy of the proposed method are acceptable. It can be seen that the determination of cysteamine using the modified electrode is effective and can be applied for their detection of cysteamine in real samples.

\section{Conclusion}

In this study, carbon-paste electrode modified with ferrocenecarboxaldehyde and $\mathrm{NiO} / \mathrm{NPs}$ was used for the determination of cysteamine in the presence of tryptophan. The electrochemical investigations showed effective electrocatalytic activity of the modified electrode in lowering the anodic overpotential for the oxidation of cysteamine and complete resolution of its anodic wave from tryptophan. A peak potential difference of $420 \mathrm{mV}$ between cysteamine and tryptophan was detected, which was large enough to determine cysteamine and tryptophan individually and simultaneously.

\section{References}

[1] M. Keyvanfard, S. Sami, H. Karimi-Maleh, and K. Alizad, "Electrocatalytic determination of cysteamine using multiwall carbon nanotube paste electrode in the presence of 3,4dihydroxycinnamic acid as a homogeneous mediator," Journal of the Brazilian Chemical Society, vol. 24, pp. 32-39, 2013.

[2] M. Yudkoff, J. W. Foreman, and S. Segal, "Effects of cysteamine therapy in nephropathic cystinosis," The New England Journal of Medicine, vol. 304, no. 3, pp. 141-145, 1981.

[3] W. A. Gahl, "Early oral cysteamine therapy for nephropathic cystinosis," European Journal of Pediatrics, Supplement, vol. 162, no. 1, pp. S38-S41, 2003.

[4] W. A. Gahl, J. G. Thoene, and J. A. Schneider, "Cystinosis: a disorder of lysosomal membrane transport," in The Metabolic and Molecular Bases of Inherited Disease, C. R. Scriver, A. L. Beaudet, W. S. Sly, and D. Valle, Eds., pp. 5085-6108, McGrawwHill, New York, NY, USA, 2001.
[5] M. Town, G. Jean, S. Cherqui et al., "A novel gene encoding an integral membrane protein is mutated in nephropathic cystinosis," Nature Genetics, vol. 18, no. 4, pp. 319-324, 1998.

[6] W. A. Gahl, F. Tietze, J. Butler DeB., and J. D. Schulman, "Cysteamine depletes cystinotic leucocyte granular fractions of cystine by the mechanism of disulphide interchange," Biochemical Journal, vol. 228, no. 3, pp. 545-550, 1985.

[7] M. Stachowicz, B. Lehmann, A. Tibi, P. Prognon, V. Daurat, and D. Pradeau, "Determination of total cysteamine in human serum by a high-performance liquid chromatography with fluorescence detection," Journal of Pharmaceutical and Biomedical Analysis, vol. 17, no. 4-5, pp. 767-773, 1998.

[8] H. Kataoka, Y. Imamura, H. Tanaka, and M. Makita, "Determination of cysteamine and cystamine by gas chromatography with flame photometric detection," Journal of Pharmaceutical and Biomedical Analysis, vol. 11, no. 10, pp. 963-969, 1993.

[9] A. J. Jonas and J. A. Schneider, "Measurement of total plasma cysteamine using high-performance liquid chromatography with electrochemical detection," Analytical Biochemistry, vol. 114, pp. 429-432, 1981.

[10] M. Keyvanfard, A. A. Ensafi, and H. Karimi-Maleh, "A new strategy for simultaneous determination of cysteamine in the presence of high concentration of tryptophan using vinylferrocene-modified multiwall carbon nanotubes paste electrode," Journal of Solid State Electrochemistry, vol. 16, no. 9, pp. 2949-2955, 2012.

[11] A. Taherkhani, H. Karimi-Maleh, A. A. Ensafi et al., "Simultaneous determination of cysteamine and folic acid in pharmaceutical and biological samples using modified multiwall carbon nanotube paste electrode," Chinese Chemical Letters, vol. 23, no. 2, pp. 237-240, 2012.

[12] C. Li, Y. Ya, and G. Zhan, "Electrochemical investigation of tryptophan at gold nanoparticles modified electrode in the presence of sodium dodecylbenzene sulfonate," Colloids and Surfaces B: Biointerfaces, vol. 76, no. 1, pp. 340-345, 2010.

[13] D. M. Reynolds, "Rapid and direct determination of tryptophan in water using synchronous fluorescence spectroscopy," Water Research, vol. 37, no. 13, pp. 3055-3060, 2003.

[14] Z. Lin, X. Chen, Z. Cai, P. Li, X. Chen, and X. Wang, "Chemiluminescence of tryptophan and histidine in $\mathrm{Ru}(\mathrm{bpy})_{3}^{2+}$ $\mathrm{KMnO}_{4}$ aqueous solution," Talanta, vol. 75 , no. 2, pp. 544-550, 2008.

[15] I. Ilisz, G. Fodor, R. Berkecz, R. Iványi, L. Szente, and A. Pétera, "Enantioseparation of $\beta$-substituted tryptophan analogues with modified cyclodextrins by capillary zone electrophoresis," Journal of Chromatography A, vol. 1216, no. 15, pp. 3360-3365, 2009. 
[16] C. Bayle, N. Siri, V. Poinsot, M. Treilhou, E. Causse, and F. Couderc, "Analysis of tryptophan and tyrosine in cerebrospinal fluid by capillary electrophoresis and "ball lens" UV-pulsed laserinduced fluorescence detection," Journal of Chromatography A, vol. 1013, no. 1-2, pp. 123-130, 2003.

[17] M. Keyvanfard, R. Shakeri, H. Karimi-Maleh, and K. Alizad, "Highly selective and sensitive voltammetric sensor based on modified multiwall carbon nanotube paste electrode for simultaneous determination of ascorbic acid, acetaminophen and tryptophan," Materials Science and Engineering C, vol. 33, no. 2, pp. 811-816, 2013.

[18] M. Fouladgar, H. Karimi-Maleh, and R. Hosseinzadeh, "Novel nanostructured electrochemical sensor for voltammetric determination of $\mathrm{N}$-acetylcysteine in the presence of high concentrations of tryptophan," Ionics, vol. 19, pp. 665-672, 2013.

[19] X. Tang, Y. Liu, H. Hou, and T. You, "Electrochemical determination of L-Tryptophan, L-Tyrosine and L-Cysteine using electrospun carbon nanofibers modified electrode," Talanta, vol. 80, no. 5, pp. 2182-2186, 2010.

[20] A. A. Ensafi and H. Karimi-Maleh, "Modified multiwall carbon nanotubes paste electrode as a sensor for simultaneous determination of 6-thioguanine and folic acid using ferrocenedicarboxylic acid as a mediator," Journal of Electroanalytical Chemistry, vol. 640, no. 1-2, pp. 75-83, 2010.

[21] A. A. Ensafi, H. Karimi-Maleh, S. Mallakpour, and B. Rezaei, "Highly sensitive voltammetric sensor based on catecholderivative-multiwall carbon nanotubes for the catalytic determination of captopril in patient human urine samples," Colloids and Surfaces B, vol. 87, no. 2, pp. 480-488, 2011.

[22] A. A. Ensafi, H. Karimi-Maleh, S. Mallakpour, and M. Hatami, "Simultaneous determination of N-acetylcysteine and acetaminophen by voltammetric method using $\mathrm{N}$ (3,4-dihydroxyphenethyl)-3,5-dinitrobenzamide modified multiwall carbon nanotubes paste electrode," Sensors and Actuators, B, vol. 155, no. 2, pp. 464-472, 2011.

[23] H. Karimi-Maleh, P. Biparva, and M. Hatami, "A novel modified carbon paste electrode based on $\mathrm{NiO} / \mathrm{CNTs}$ nanocomposite and (9, 10-dihydro-9, 10-ethanoanthracene-11, 12-dicarboximido)4-ethylbenzene-1, 2-diol as a mediator for simultaneous determination of cysteamine, nicotin amide adenine dinucleotide and folic acid," Biosensors and Bioelectronics, vol. 48, pp. 270275, 2013.

[24] T. Tavana, M. A. Khalilzadeh, H. Karimi-Maleh, A. A. Ensafi, H. Beitollahi, and D. Zareyee, "Sensitive voltammetric determination of epinephrine in the presence of acetaminophen at a novel ionic liquid modified carbon nanotubes paste electrode," Journal of Molecular Liquids, vol. 168, pp. 69-74, 2012.

[25] S. Salmanpour, T. Tavana, A. Pahlavan et al., "Voltammetric determination of norepinephrine in the presence of acetaminophen using a novel ionic liquid/multiwall carbon nanotubes paste electrode," Materials Science and Engineering C, vol. 32, pp. 1912-1918, 2012.

[26] H. Beitollah, M. Goodarzian, Khalilzadeh et al., "Electrochemical behaviors and determination of carbidopa on carbon nanotubes ionic liquid paste electrode," Journal of Molecular Liquids, vol. 173, pp. 137-143, 2012.

[27] J.-B. Raoof, R. Ojani, and H. Karimi-Maleh, "Carbon paste electrode incorporating 1-[4-(ferrocenyl ethynyl) phenyl]-1ethanone for electrocatalytic and voltammetric determination of tryptophan," Electroanalysis, vol. 20, no. 11, pp. 1259-1262, 2008.
[28] M. Elysi and M. A. Khalilzadeh, "High sensitive voltammetric sensor based on pt/CNTs nanocomposite modified ionic liquid carbon paste electrode for deter, mination of Sudan I in food samples," Food Chemistry, vol. 141, pp. 4311-4317, 2013.

[29] E. Afsharmanesh, H. Karimi-Maleh, A. Pahlavan, and J. Vahedi, "Electrochemical behavior of morphine at $\mathrm{ZnO} / \mathrm{CNT}$ nanocomposite room temperature ionic liquid modified carbon paste electrode and its determination in real samples," Journal of Molecular Liquids, vol. 181, pp. 8-13, 2013.

[30] H.J. Jeon, S.C. Yi, and S.G. Oh, "Preparation and antibacterial effects of $\mathrm{Ag}-\mathrm{SiO}_{2}$ thin films by sol-gel method," Biomaterials, vol. 24, no. 27, pp. 4921-4928, 2003.

[31] A. A. Ensafi, E. Khoddami, B. Rezaei, and H. Karimi-Maleh, "P-Amiophenol -multiwall carbon nanotube-Tio 2 electrode as a sensor for simulations determination of pencillamine and uric acid," Colloid and Surface B, vol. 81, pp. 42-29, 2010.

[32] H. Beitollahi, H. Karimi-Maleh, and H. Khabazzadeh, "Nanomolar and selective determination of epinephrine in the pre sence of norepinephrine using carbon paste electrode modified with carbon nanotubes and novel 2-(4-oxo-3-phenyl-3,4dihydroquinazolinyl)- $\mathrm{N}^{\prime}$ - phenylhydrazinecarbothioamide," Analytical Chemistry, vol. 80, no. 24, pp. 9848-9851, 2008.

[33] M. Roodbari Shahmiri, A. Bahari, H. Karimi-Maleh, R. Hosseinzadeh, and N. Mirnia, "Ethynylferrocene-NiO/MWCNT /MWCNT nanocomposite modified carbon paste electrode as a novel voltammetric sensor for simultaneous determination of glutathione and acetaminophen," Sensors and Actuators B: Chemical, vol. 177, pp. 70-77, 2013.

[34] R. Moradi, S. A. Sebt, H. Karimi-Maleh et al., "Synthesis and application of FePt/CNTs nanocomposite as a sensor and novel amide ligand as a mediator for simultaneous determination of glutathione, nicotinamide adenine dinucleotide and tryptophan", Physical Chemistry Chemical Physics, vol. 15, no. 16, pp. 5888-5897, 2013.

[35] M. Bijad, H. Karimi-Maleh, and M. A. Khalilzadeh, "Application of $\mathrm{ZnO} / \mathrm{CNT}$ nanocomposite ionic liquid paste electrode as a sensitive voltammetric sensor for determination of ascorbic acid in food samples," Food Analytical Methods, 2013.

[36] M. Fouladgar, H. Karimi-Maleh, and R. Hosseinzadeh, "Novel nanostructured electrochemical sensor for voltammetric determination of $\mathrm{N}$-acetylcysteine in the presence of high concentrations of tryptophan," Ionics, vol. 19, no. 4, pp. 665-672, 2013.

[37] H. Karimi-Maleh, A. A. Ensafi, and H. R. Ensafi, "Ferrocenedicarboxylic acid modified carbon paste electrode: a sensor for electrocatalytic determination of hydrochlorothiazide," Journal of the Brazilian Chemical Society, vol. 20, no. 5, pp. 880-887, 2009.

[38] Z. Galus, Fundumentals of Electrochemical Analysis, Ellis Horwood, New York, NY, USA, 1976.

[39] A. A. Ensafi and H. Karimi-Maleh, "A voltammetric sensor based on modified multiwall carbon nanotubes for cysteamine determination in the presence of tryptophan using paminophenol as a mediator," Electroanalysis, vol. 22, no. 21, pp. 2558-2568, 2010. 

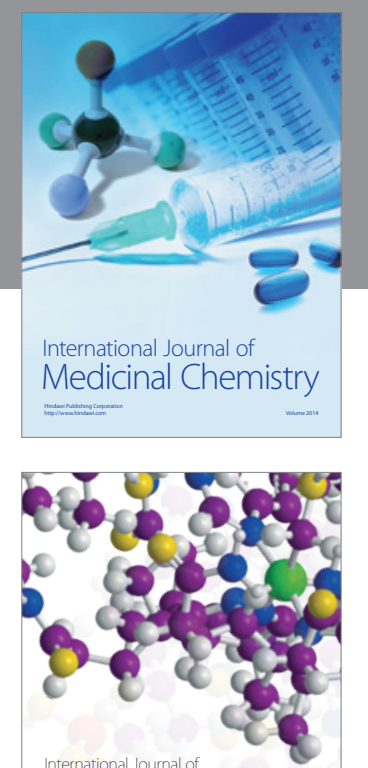

\section{Carbohydrate} Chemistry

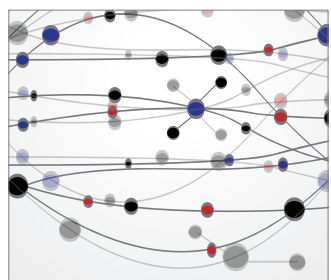

The Scientific World Journal
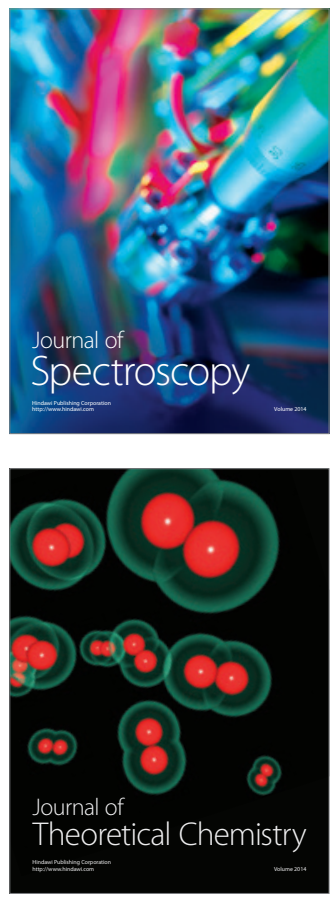
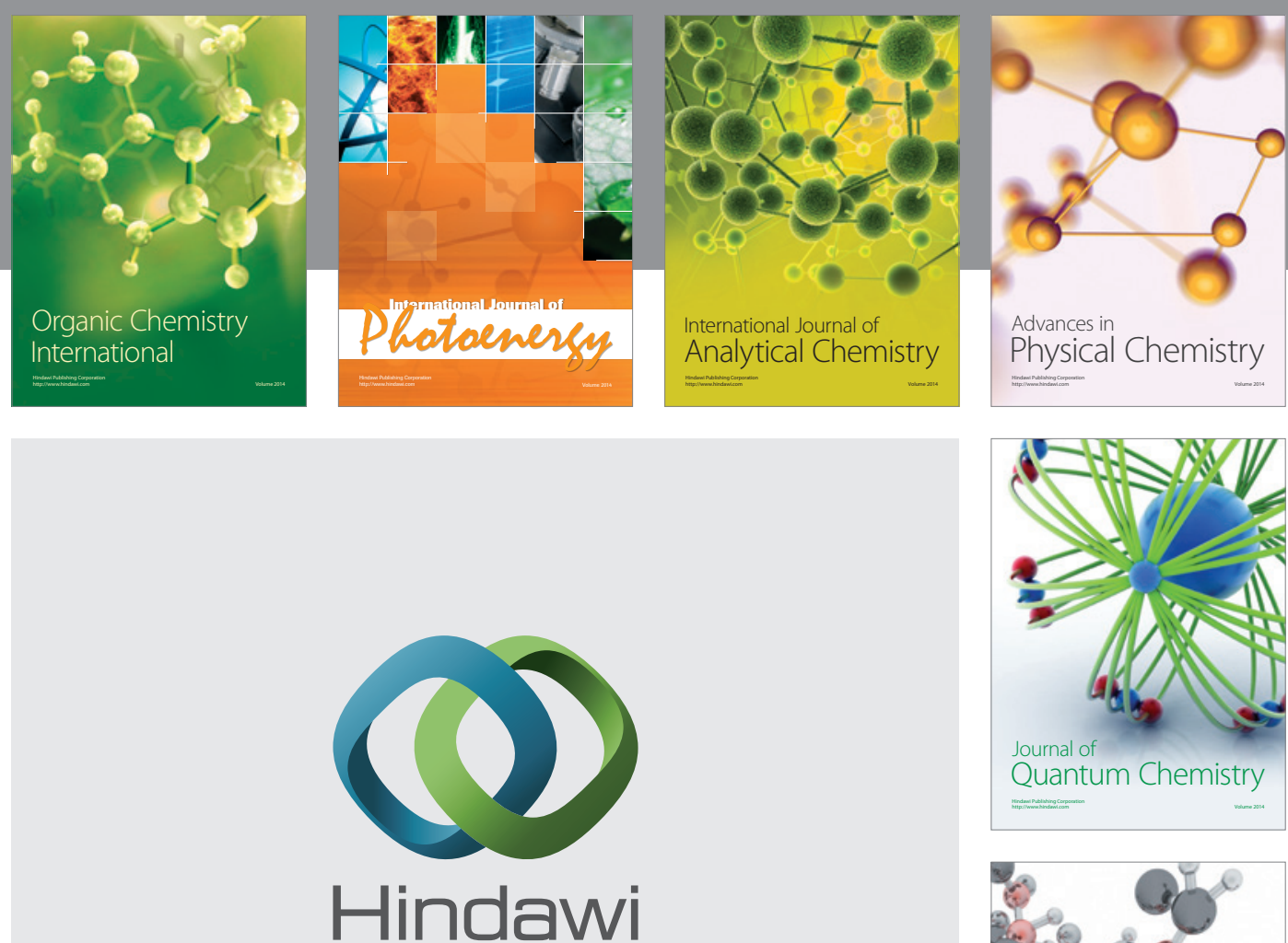

Submit your manuscripts at

http://www.hindawi.com

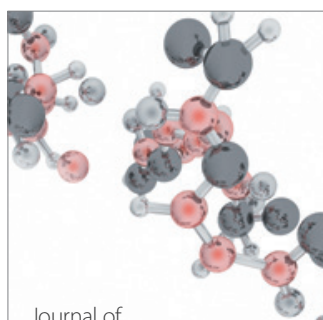

Analytical Methods

in Chemistry

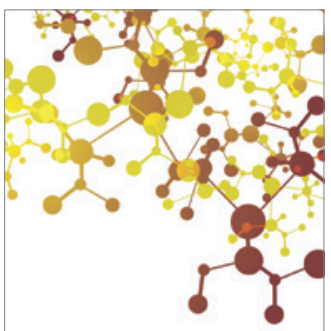

Journal of

Applied Chemistry

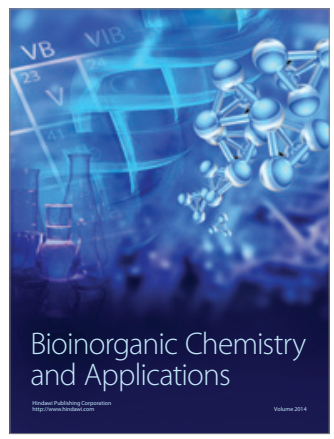

Inorganic Chemistry
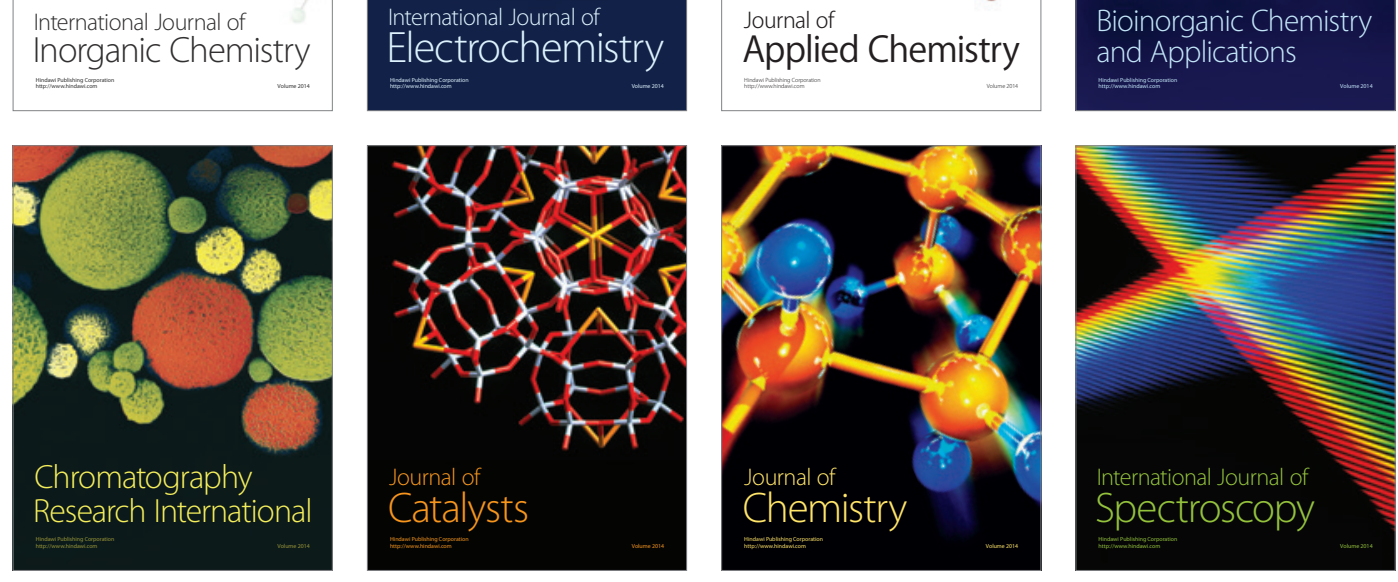